\title{
BMJ Open The PACT study protocol: a time series study investigating the impact, acceptability and cost of an integrated model for psychosocial screening, care and treatment of patients with urological and head and neck cancers
}

\author{
Afaf Girgis, ${ }^{1}$ Brian Kelly, ${ }^{2,3}$ Allison Boyes, ${ }^{4}$ Marion Haas, ${ }^{5}$ Rosalie Viney, ${ }^{5}$ \\ Joseph Descallar, ${ }^{1}$ Hayley Candler, ${ }^{1}$ Douglas Bellamy, ${ }^{6}$ Anthony Proietto ${ }^{7}$
}

To cite: Girgis A, Kelly B, Boyes $A$, et al. The PACT study protocol: a time series study investigating the impact, acceptability and cost of an integrated model for psychosocial screening, care and treatment of patients with urological and head and neck cancers. BMJ Open 2014:4:e004147.

doi:10.1136/bmjopen-2013004147

- Prepublication history for this paper is available online. To view these files please visit the journal online (http://dx.doi.org/10.1136/ bmjopen-2013-004147).

The PACT Study team also includes Catherine Adams, Debbie O'Brien, Gai Shylan, Ben Britton, Sandy Grant, Vicky Parker, Chris Wratten and Deanna Sue.

Received 29 September 2013 Revised 30 November 2013 Accepted 4 December 2013

\section{CrossMark}

For numbered affiliations see end of article.

Correspondence to Professor Afaf Girgis; afaf.girgis@unsw.edu.au

\section{ABSTRACT}

Introduction: While there is good evidence of the effectiveness of a variety of interventions and services to prevent and/or relieve distress experienced by people affected by cancer, much of this psychosocial morbidity is undetected and untreated, with consequent exacerbated suffering, decreased satisfaction with care, impaired adherence to treatment regimens and poorer morbidity and mortality outcomes. The objective of this study is to develop, implement and assess the impact, acceptability and cost of an integrated, patient-centred Psychosocial Assessment, Care and Treatment (PACT) model of care for patients with urological and head and neck cancers.

Methods and analysis: A time series research design will be used to test the PACT model of care, newly introduced in an Australian tertiary hospital. The primary outcome is system-level impact, assessed through audit of patients' medical records and Medicare claims for follow-up care. The secondary outcomes are impact of the model on patients' experience and healthcare professionals' (HCPs) knowledge and confidence, assessed via patient and HCP surveys at baseline and at followup. Acceptability of the intervention will be assessed through HCP interviews at follow-up, and cost will be assessed from Medicare and Pharmaceutical Benefits Scheme claims information and information logged pertaining to intervention activities (eg, time spent by the newly appointed psycho-oncology staff in direct patient contact, providing training sessions, engaging in case review) and their associated costs (eg, salaries, training materials and videoconferencing).

Ethics and dissemination: Ethics approval was obtained from the Human Research Ethics Committees of Hunter New England Local Health District and the University of NSW.

Results: The results will be widely disseminated to the funding body and through peer-reviewed

\section{Strengths and limitations of this study}

- The study has been developed specifically to address the existing gaps in psychosocial care, and proposes a model of care which will be integrated, high quality, evidence-based, embedded in routine practice and responsive to individual patients' needs.

- It promotes an active role for frontline staff, as well as improved coordination and continuity of care, particularly for patients in rural and remote areas.

- The lack of research attention on patients with urological or head and neck cancers, despite their burden on the Australian community, is addressed.

- The translational capacity of the programme is enhanced through the support of a very strong collaborative team, a strong methodology for health services research (including cost analyses, which are often overlooked in interventional research) and a strong support for the integration of psychosocial care into routine care. Importantly, this work has a substantial potential for translation into other cancer services, beyond the current study.

- The target population is vulnerable and experiencing an acute stressor that may impact on recruitment.

- As the intervention will continue over a 24-month period, changes in health professional staff over that time may be substantial. Consequently, some of the health professionals who complete a survey at 24 months may be only minimally exposed to the intervention and have diluted perceptions of impact.

- Other health initiatives may be introduced to hospitals in the study area, which could affect the impact of this intervention.

publications, HCP and consumer publications, oncology conferences and meetings.

Trial registration: The study is registered with the Australian New Zealand Clinical Trials Registry with registration number ACTRN12613000916741. 
Emotional distress, pain and fatigue are commonly experienced by the majority of patients with cancer, while other issues are unique to specific cancer types. Individuals diagnosed with urological cancers (bladder, kidney, prostate, testicles, penis) typically experience urinary and bowel dysfunction and sexual problems. ${ }^{1}$ Those diagnosed with head and neck $(\mathrm{H} \& \mathrm{~N})$ cancers (mouth, jaw, throat, larynx, salivary glands, skin of H\&N including melanoma, thyroid) often experience profound disfigurement and functional disability, changed body image, speech difficulties, nutritional problems and have higher suicide rates than other people with cancer. ${ }^{2}$ Patients with urological and H\&N cancer often report negative changes in their intimate and social relationships. ${ }^{1}{ }^{3}$ Some cancers, including urological and $\mathrm{H} \& \mathrm{~N}$ cancers, are more prevalent in rural areas, ${ }^{4}$ where people experience major difficulties accessing cancer services, including psychosocial care. ${ }^{5}$ There is good evidence of the effectiveness of a variety of interventions and services to prevent and/or relieve the distress experienced by patients with cancer. ${ }^{6}$ Nevertheless, it is well established that most of the psychosocial morbidities experienced by patients with cancer are undetected and untreated. ${ }^{7}$ Failure to address these problems can exacerbate suffering and lead to decreased patient satisfaction with care, impaired adherence to treatment regimens and poorer morbidity and mortality outcomes. ${ }^{8}$

Recent cancer patient satisfaction surveys in Australia, the UK and Canada have highlighted the problems in coordination of services and limitations in psychosocial care. ${ }^{9-11}$ Skills, confidence and beliefs of clinical staff regarding psychosocial aspects of care are important contributing factors. ${ }^{12}$ Many healthcare professionals (HCPs) are not aware of effective evidence-based strategies to address patients' concerns or underestimate the benefits of attending to psychosocial needs or referring to psychosocial personnel or services. There are a few formalised mechanisms for communication between healthcare providers and systems of care that may contribute to patients receiving fragmented and poorly coordinated care, especially those who reside in rural areas. ${ }^{8}$ With well-supported stepped care models, the needs of the majority of patients with cancer can be met without referral to specialist psychosocial services. ${ }^{13}$ Models of care that provide basic psychosocial care delivered by frontline healthcare providers (eg, oncology nurses), with appropriate training and mentorship by psycho-oncology specialists, have demonstrated efficacy and cost-effectiveness in terms of the increase in quality-adjusted life-years achieved. ${ }^{14}$

The Institute of Medicine ${ }^{8}$ has recommended a model for integrated psychosocial cancer care that comprises: (1) identifying patients' health needs through screening and assessment; (2) linking patients to health services via structured referral, case management and clinical integration of services; (3) supporting patients in illness self-management; (4) coordinating psychosocial and biomedical healthcare through care coordinators, multidisciplinary team meetings, multidisciplinary care plans and electronic health records and (5) following up on care delivery by telephone calls or web-based technology to re-evaluate and adjust the patient's care plan. ${ }^{8}$ Achieving such a model of care in most specialist oncology services and evaluating its effectiveness represent major challenges. ${ }^{15} 16$ These challenges are accentuated in settings where cancer care is integrated into general medical or surgical care, with the absence of dedicated on-site cancer teams. This means that staff may not identify themselves as cancer clinicians and service models are generic to cater for a diverse range of illness groups. Nevertheless, this represents the setting in which a significant proportion of patients with cancer experience at least part of their treatment. Within this type of care delivery setting, how can effective models of integrated psycho-oncology care be developed, implemented and evaluated? This is one of the key questions this project aims to investigate.

\section{OBJECTIVE}

The objective of this study is to develop, implement and assess the impact, acceptability and cost of an integrated, patient-centred model for psychosocial screening, care and treatment of patients with urological and $\mathrm{H} \& \mathrm{~N}$ cancers at a large tertiary referral hospital.

\section{STUDY DESIGN}

A time series research design will be utilised to test the Psychosocial Assessment, Care and Treatment (PACT) model of care (detailed below). While the RCT is often used as the gold standard for assessing the effectiveness of health interventions, it is not always practical in health services research. A time series design will be used, as it is regarded as the strongest quasi-experimental design for evaluating longitudinal effects of interventions ${ }^{17}$ and is an acceptable design for inclusion in Cochrane reviews. ${ }^{18}$ Time series designs attempt to detect whether an intervention has an effect significantly greater than the underlying secular trend, ${ }^{18}$ and are useful in quality improvement research for evaluating the effects of interventions when it is difficult to randomise patients. The study will focus on system-level outcomes as being of primary interest. We will monitor the process, outcomes and costs of establishing the specialised psycho-oncology service, including the development of evidence-based management protocols and referral pathways specifying defined roles for different health professionals within the cancer care setting. Table 1 provides an overview of the study design and time line.

\section{METHODS}

\section{Study setting}

The setting for this study is John Hunter Hospital $(\mathrm{JHH}) /$ Royal Newcastle Centre, the largest tertiary referral teaching hospital in the Hunter New England Local Health District (HNELHD) of New South Wales 
Table 1 Study design and data collection timeline

\begin{tabular}{|c|c|c|}
\hline Time period & Data collection & Intervention delivery \\
\hline \multirow[t]{3}{*}{$\begin{array}{l}\text { Starting at } \\
0 \text { months } \\
\text { (March 2013) }\end{array}$} & $\begin{array}{l}\text { Recruitment of baseline HCP sample } \\
\text { Completion of Health Professional Knowledge and } \\
\text { Confidence Survey }\end{array}$ & \\
\hline & $\begin{array}{l}\text { Recruitment of Patient Cross-sectional Sample \#1 } \\
\text { Completion of Patient Experience Survey }\end{array}$ & $\begin{array}{l}\text { Development and delivery of communication skills } \\
\text { training to health professionals }\end{array}$ \\
\hline & $\begin{array}{l}\text { Retrospective audit of medical and hospital records } \\
\text { for consenting patients }\end{array}$ & $\begin{array}{l}\text { PACT intervention delivery throughout the study } \\
\text { period }\end{array}$ \\
\hline $\begin{array}{l}\text { Starting at } \\
12 \text { months }\end{array}$ & $\begin{array}{l}\text { Recruitment of Patient Cross-sectional Sample \#2 } \\
\text { Completion of Patient Experience Survey } \\
\text { Retrospective audit of medical and hospital records } \\
\text { for consenting patients }\end{array}$ & $\begin{array}{l}\text { Ongoing monitoring of costs of the intervention (time } \\
\text { spent in direct patient contact, on staff training, } \\
\text { interprofessional case reviews and other } \\
\text { communications required to support rural and } \\
\text { regional providers) }\end{array}$ \\
\hline \multirow{13}{*}{$\begin{array}{l}\text { Starting at } \\
24 \text { months }\end{array}$} & Recruitment of Patient Cross-sectional Sample \#3 & \\
\hline & Completion of Patient Experience Survey & \\
\hline & $\begin{array}{l}\text { Retrospective audit of medical and hospital records } \\
\text { for consenting patients }\end{array}$ & \\
\hline & Recruitment of postintervention HCP sample & \\
\hline & Completion of Health Professional Knowledge and & \\
\hline & Confidence Survey & \\
\hline & Recruitment and interviews with purposively sampled & \\
\hline & HCPs regarding acceptability of the PACT intervention & \\
\hline & $\begin{array}{l}\text { Retrospective review of Medicare and PBS claims } \\
\text { data for use and costs of medical services and }\end{array}$ & \\
\hline & pharmaceuticals for the three cross-sectional patient & \\
\hline & $\begin{array}{l}\text { samples (Department of Human Services data } \\
\text { extraction) }\end{array}$ & \\
\hline & Review of hospital's databases & \\
\hline & $\begin{array}{l}\text { Assessment of set-up and on-going costs associated } \\
\text { with the PACT intervention }\end{array}$ & \\
\hline
\end{tabular}

(NSW), Australia. It provides the main services for a large sector of the state of NSW, comprising a population of over 850000 people, including a major metropolitan centre and several large regional centres, as well as many smaller rural centres and remote communities. This area has an average of 4171 new cases of cancer diagnosed per annum, with over 9000 inpatient separations per year for cancer-related conditions. ${ }^{19}$ It is the main teaching hospital of the University of Newcastle.

The current model of cancer care at JHH, the site for this study, includes investigation, diagnosis, surgery and follow-up surveillance. Patients needing radiation or chemotherapy are referred to a nearby specialist cancer service and generally return to $\mathrm{JHH}$ or a regional hospital facility for follow-up and monitoring. In 2012, 124 urology and $69 \mathrm{H} \& \mathrm{~N}$ patients received inpatient cancer care at JHH. The JHH urology service comprises five senior urologists and four dedicated outpatient nursing staff, and the H\&N unit comprises four senior surgeons, one dedicated outpatient nurse and four ward-based nurses. Each inpatient unit comprises 20 nursing staff caring for patients with a range of cancer and noncancer conditions. For both tumour groups, multidisciplinary team meetings provide a forum to discuss individuals with complex cancers. Although each unit is supported by dedicated allied health clinicians, JHH has no dedicated psycho-oncology services. Patients requiring psycho-oncology assessment are referred either to generic Liaison Psychiatry, which provides a limited role including advice, inpatient consultation and a restricted level of outpatient assessment, or to the psycho-oncology service at the nearby cancer hospital, which is a separate service with no shared records and located at a different site to patients' routine outpatient care at $\mathrm{JHH}$.

\section{Eligibility criteria}

Patients

Inclusion criteria are: (1) aged 18 years or over, (2) diagnosed with a urological cancer or an $\mathrm{H} \& \mathrm{~N}$ cancer, and (3) receiving inpatient and/or outpatient care at JHH. Patients from metropolitan and all rural areas served by this hospital are included in the study.

\section{Staff}

Staff will be eligible to complete the Knowledge and Confidence Survey if they: (1) are a nursing or allied health staff member and (2) provide care for patients who are receiving inpatient or outpatient urological or $\mathrm{H} \& \mathrm{~N}$ cancer services at this site. Staff will be eligible to 
participate in interviews to assess the acceptability of the PACT care model if they meet the following inclusion criteria: they (1) are a medical, nursing or allied health staff member, (2) provide care for patients who are receiving inpatient or outpatient urological or $\mathrm{H} \& \mathrm{~N}$ cancer services and (3) have been involved in the PACT care pathway of one or more patients, either on-site at $\mathrm{JHH}$, or through provision of follow-up care, following discharge from JHH.

\section{Intervention}

It is important to note that this study focuses on a system-level intervention aimed at modifying provision of service to all patients attending these units, rather than recruiting a sample of patients for the provision of a specific psychosocial intervention. While data are collected periodically from patients attending this hospital and from staff, this is chiefly with the intention of examining the impact of the service changes. The PACT model focuses on a system of care for patients with H\&N or urological cancers. This model aims to systematise the approach to screening for distress and responding to that distress in a coordinated manner, including facilitating continuity of care for patients who reside some distance from the acute care setting where they were treated for cancer. In order to address the needs of those patients in rural regions, a specific component of the innovation introduced to the system of care is extended to this population. The development of this care model includes the following key components:

1. The implementation for inpatients and outpatients of the two surgical units of routine screening for distress, and associated psychosocial care plans.

2. The identification of intervention options for all levels of need, and pathways to specialist psychooncology care if required.

3. The addition of dedicated psycho-oncology clinical services (including psychologist, psychiatrist and mental health nurse).

4. The provision of staff development and support to implement such a model (including training in skilled communication to identify and respond to emotional distress), and structured case review for complex or challenging cases. The latter strategies will incorporate oncology clinical staff and others working with patients with cancer within urban, regional and remote communities.

5. Videoconferencing to facilitate case review meetings between clinicians at the hospital base site and those at rural sites who are engaged in the ongoing postdischarge care of patients initially treated at the hospital.

In order to achieve these intervention goals, the following are intrinsic to the service model:

A. A dedicated Clinical Nurse Consultant (CNC), with experience in psycho-oncology and adult education, will coordinate the establishment and delivery of the model, with a special focus on development and implementation of a systematic and structured approach to routine screening, triage and management of individual physical, psychological and social concerns during active treatment and at follow-up for patients with cancer.

B. The CNC, a newly appointed Clinical Psychologist and two of the investigators with experience in communication skills training (AG, BK) will develop and deliver a communication skill-based training programme to support frontline clinical staff (mostly nursing and allied HCPs) in implementing the psycho-oncology care model and facilitating within-team communication to enhance continuity of patient care. This training programme will be run on numerous occasions to reach as many frontline staff as possible during the intervention period and is based on an evidence-based consultation skills training programme previously developed and evaluated by our team. ${ }^{20} 21$

C. The CNC and Clinical Psychologist will facilitate the delivery of the care model through the training of frontline staff, provision of clinical assessment and specialised evidence-based care for patients who are referred to the psycho-oncology service, monitoring of progress on psychosocial care plans including with rural clinicians through case reviews, and providing advice as required to rural clinicians to support linking patients to local rural specialised services. Where specialised services are not available, the Clinical Psychologist will collaborate with the local clinician (eg, rural clinical nurse) to provide outreach specialist assessment by videoconference. The lead psychiatrist (BK) will provide clinical oversight of the programme, participate in staff training and direct clinical evaluation and treatment of patients with the highest level of distress or complexity.

Clinical practice guidelines recommend routine distress screening of patients with cancer (with feedback to healthcare providers) at periods of increased vulnerability to ensure that those at risk are identified promptly and offered appropriate treatment. ${ }^{22}$ As part of the newly developed model of care, all inpatient and outpatient patients with urology and $\mathrm{H} \& \mathrm{~N}$ cancer will be screened at their first diagnostic or treatment visit and at each subsequent follow-up visit, using the distress thermometer (DT) and accompanying Problem Checklist, ${ }^{13}$ which will inform the development of a care plan to address the issues identified through the screening and second-line inquiry. The care plan will facilitate provision of care tailored to the specific needs of patients and promote continuity of care across care settings and providers, including with HCPs in the rural and regional areas.

The screening and problem checklist will act as a trigger for frontline staff to inquire about, and discuss the cause/s of, distress with patients whose distress levels are above the recommended cut-off of 4 or more out of $10{ }^{23}$ Training for frontline staff will focus on discussing the cause/s of the distress, developing a psychosocial 
care plan to address identified concerns providing information and/or basic counselling, or referring patients with significant or persistent distress to the psycho-oncology service and facilitating continuity of care, including linking patients with hospital and community services as required. Staff will also assist patients with access to self-management information on tablets which will be available during their hospital visit. Long-term sustainability of these service changes will be promoted through organisational leadership and management support, engagement of cancer clinician leaders in each unit in the design and evaluation of the service model, and development of a model with the aim of adaptability and flexibility to diversity of locations and patient complexity (eg, through complex case review and revision of psychosocial care plans and active involvement of clinicians from rural locations in this process). ${ }^{24}$

\section{Outcomes}

The primary outcome is a system-wide increase in the proportion of eligible patients receiving care at the study facility who complete a DT and accompanying Problem Checklist (see Data Collection Methods) on at least one occasion and have a psychosocial care plan developed which complies with recommended care pathways. This outcome will be assessed at baseline, then at 12 and 24 months postbaseline (ie, following the establishment of the new model of care-refer to Intervention section).

The secondary outcomes are: (1) an increase in the proportion of eligible patients receiving care at the study facility who report positive experiences of their cancer care at 12 and 24 months postbaseline compared with patients receiving care in this facility at baseline and (2) an increase in the proportion of health professionals providing care to the eligible patient population at the study facility who report high levels of knowledge and confidence in responding to patients' psychosocial concerns at 24 months postbaseline compared with the health professionals caring for the eligible patient population at baseline. HCP satisfaction with the PACT intervention will also be assessed via interviews at follow-up, and the resource use and costs of the intervention will be monitored by maintaining comprehensive logs of intervention activities (eg, time spent by the CNC and Clinical Psychologist in direct patient contact, providing training sessions, engaging in case review) and ascribing the associated costs (eg, CNC and Clinical Psychologist salaries and on-costs, materials used in training sessions, videoconferencing costs).

\section{Recruitment}

Patients

Three cross-sectional samples of the current inpatients and outpatients will be recruited, at baseline, 12 and 24 months, to complete a Patient Experience Survey (of their cancer care) and provide consent for access to their hospital and other medical records. The main purpose of these data collection phases is the evaluation of the impact of system-level changes, rather than recruiting patients who will undertake the intervention. During the study audit periods at baseline, 12 and 24 months, the Research Officer will contact staff of the outpatient clinics and inpatient wards in which care is provided for urological and H\&N cancers, on a weekly basis, to identify whether patients meeting the inclusion criteria will be attending those clinics or wards that week. The Research Officer will attend those clinics and/or wards at which the potential participants will be present, briefly introduce those patients to the study, answer questions and provide interested persons with an Information Pack. The Information Pack will contain an Information Letter, a Consent Form for data to be obtained from HNELHD, a Consent Form for data to be obtained from the Department of Human Services, a Request for Summary of Study Results Form, a copy of the self-administered Patient Experience Survey and a Paperwork Return Checklist. Patients will be asked to take the Information Pack home to consider before completing the survey and consent forms and posting them back to the researchers, using a self-addressed reply paid envelope, within 10 days. Staff at the outpatient clinics and inpatient wards will also be supplied with Information Packs to distribute to eligible patients who attend the clinic when the Research Officer is not in attendance.

\section{Staff}

Health professional knowledge and confidence survey

Two cross-sectional samples of HCPs involved in the care of patients with H\&N or urological cancers at the study facility will be recruited during the study audit periods at baseline and at 24 months, to assess the skills development of clinical staff who work at the parent facility. At each time point, the Research Officer will contact the Managers of the inpatient wards and outpatient clinics in which care is provided to patients with urological and $\mathrm{H} \& \mathrm{~N}$ cancers. The Managers will be asked to identify the nursing and allied health staff members who routinely provide care to the patients of their respective wards/clinics, as well as each staff member's employment status as either permanent or casual. A list of HCPs eligible to receive an Information Pack will then be generated, and Information Packs will be sent via internal mail to the department at which each staff member is based. The Information Pack will contain an Information Letter and a copy of the self-administered Knowledge and Confidence Survey for HCPs to complete and post back to the researchers, using a selfaddressed reply paid envelope, within 10 days. A second survey will be mailed to HCPs who do not return a completed survey within 4-6 weeks and a third survey will be sent to non-returnees 4-6 weeks after that.

\section{Health professional receptivity and acceptability interviews}

At approximately 24 months (nearing study completion), purposively sampled allied health, nursing and medical 
staff will be interviewed by the Research Officer about the acceptability of the key aspects of the PACT integrated model of psychosocial care (including screening, triage, access to psychosocial services/providers, clinical case reviews), perceived effectiveness of the model at improving care and perceived impact of the staff training. The Research Officer will send an Information Pack to those staff members who meet the inclusion criteria. The Information Pack will contain an Information Letter, Consent Form and Request for Interview Transcript Form. Consenting staff will participate in a 20 min telephone interview at a mutually convenient time.

\section{Data collection methods}

The primary outcome of system-level change will be assessed through audit of patient medical records and through Medicare and Pharmaceutical Benefits Scheme (PBS) claims information for follow-up care sought via referral through the new model of care; the secondary outcomes of impact of the model on patient experiences and on HCP knowledge and confidence will be assessed via patient and HCP surveys. Acceptability of the intervention will be assessed via HCP interviews, and cost of the intervention will be assessed from information collected through Medicare and PBS claims information, as well as information logged pertaining to intervention activities (eg, time spent by the $\mathrm{CNC}$ and Clinical Psychologist in direct patient contact, providing training sessions, engaging in case review) and their associated costs (eg, CNC and Clinical Psychologist salaries and on-costs, materials used in training sessions, videoconferencing costs).

\section{Medical records audit and Medicare and PBS claims information}

At baseline, 12 and 24 months, the files of all patients who provide their consent will be reviewed by the Research Officer and an appointed research assistant who is not involved in the intervention delivery, to calculate the proportions of patients (1) who have completed a DT and accompanying Problem Checklist at least once, (2) who have had a psychosocial care plan developed and (3) whose management, including referrals, complies with recommended care pathways. A checklist will be used to achieve a systematic approach to extraction of these records. The coders (Research Officer and a second research assistant) will initially review one file with the Clinical Psychologist to ensure consistency in understanding of the checklist and $10 \%$ of the files will be double-coded by the two coders to calculate interrater reliability.

Medicare and PBS claims information will also be extracted by the Department of Human Services for those participants who provide their consent, and will be reviewed by the health economist investigators $(\mathrm{MH}$ and $\mathrm{RV})$ to extract information relating to psychosocial care delivered in a hospital or community health setting, by a private provider, or by a general practitioner.
Patient experience survey

A 35-item survey will include items assessing patient perceptions of care received, relating to the dimensions of emotional support, information, education and coordination of care. All items are phrased from the first-person perspective (eg, "I had confidence and trust in the staff treating me"), to be answered using a five-point Likert-type scale ranging from 1='strongly disagree' to $5=$ 'strongly agree'. A patient experience score will be aggregated based on the sum of selected responses. The survey contains three items from the Cancer Care Survey $^{25}$ and eight items from the Hospital CareOvernight Patient Survey ${ }^{26}$; all adapted such that they could be answered from the first-person perspective. The survey also contains 10 items from the Critical Cancer Care Events Scale, ${ }^{27}$ some of which required adaptation so that they referred to a broader group of health professionals than doctors, and all of which were adapted to be answered on a five-point Likert-type scale (as described above).

The survey will also measure key sociodemographic, disease and medical variables, including age, residential location, gender, marital status, indigenous identification, languages spoken, country of birth, educational attainment, employment status, private health coverage, income, cancer type, date of diagnosis, treatment received, recurrence status and number of prior inpatient admissions and outpatient clinic visits.

\section{Health professional knowledge and confidence survey}

A 65-item survey will include items targeting health professionals' knowledge, skills and confidence pertaining to responding to patients' specific psychosocial concerns. The survey contains 12 items from a Confidence in Communication Skills and Discussing Prognosis and End-of-Life Issues module used by Clayton et al. ${ }^{28}$ The survey also contains a case study and associated Care Planning, Monitoring and Review items from the Client-Centred Care-Training Needs Survey ${ }^{29}$; all adapted such that they refer to a patient with urological cancer and explicitly address psychosocial care. A Clinician Belief Scale is contained in the survey; based on the Physician Belief Scale, ${ }^{30}$ it contains all 32 items of the Physician Belief Scale, but has been renamed to apply to a broader group of health professionals than doctors, and items will be answered on a five-point Likert-type scale ranging from $1=$ 'strongly disagree' to $5=$ 'strongly agree'. Finally, the survey contains the single-item Clinician Burnout survey, which is the Physician Burnout survey, ${ }^{31}$ renamed to apply to a broader group of health professionals than doctors. A knowledge/ confidence score will be aggregated based on the sum of selected responses.

The survey will also measure key sociodemographic, experience and training variables, including age, residential location, gender, occupational specialty, the number of years of experience in (1) their current specialty and (2) in cancer care, the number of hours spent weekly in direct patient contact, indigenous 
identification, languages spoken, country of birth and country of training.

\section{Health professional receptivity and acceptability interview}

An interview will be scheduled with consenting participants to assess the acceptability of the key aspects of the integrated model of psychosocial care (including screening, triage, access to psychosocial services/providers and clinical case reviews), perceived effectiveness of the model in improving care and the perceived impact of training. It is anticipated that the interview will take $20 \mathrm{~min}$, with an audio recording being made for transcription purposes. An interview schedule previously developed by our team has been adapted for use in the present study, and examples of questions in the interview guide include: "Have you been aware of there being a more systematic approach to the provision of psychosocial care for people with H\&N or urological cancers over the past year or so compared with previously?" and "What's your impression of patient/family member/caregiver attitudes towards the new model (eg, acceptance)?”

\section{Intervention costs}

In keeping with the time series design, information about resource use and associated costs will be obtained at baseline, 12 and 24-month time points. Information will be obtained from the hospital's databases regarding emergency department (ED) attendance, triage category, whether the patient was admitted or discharged from the ED and their diagnosis. Information about use and costs of medical services and pharmaceuticals will be obtained from Medicare and PBS claims data. Specific set-up and ongoing costs associated with staff training, interprofessional case reviews and other communications required to support rural and regional providers will also be monitored.

\section{Sample size}

\section{Patients}

Data from the initial 25 patient surveys completed were used to estimate the required patient sample size. The mean patient experience score at baseline was rated highly at 45 of a possible 55 . Hence, the research team determined that a five-point improvement between baseline and the last follow-up (24 months) would be a meaningful change, assuming a SD of 7.58 (derived from current surveys), significance level of $5 \%$ and power of $80 \%$, with at least 38 patients needing to be recruited at each time point to detect this change over time.

\section{Healthcare professionals}

Data from the first 28 surveys completed were used to estimate the required sample size. The mean score of knowledge/confidence was moderate at 61.5 of a possible 105 . Hence, it was determined that at least 48 health professionals were required to be recruited at each time point to achieve a significant improvement of 10 points between baseline and follow-up (24 months), assuming a SD of 17.13 (derived from current surveys), significance level of $5 \%$ and power of $80 \%$.

\section{Data management}

Data which are collected in paper format (ie, patient consent forms, surveys and requests for study results, and HCP surveys, requests for study results and interview transcripts) will be stored in a locked cabinet, accessible only by the Research Officer. Data which are collected in computer file format (ie, data obtained through the Department of Human Services and HNELHD and interview audio and transcript files) will remain in computer file format. In addition, computer files will be created for the entry and storage of participant details and survey responses. All of these electronically stored data will be maintained in separate, password-protected files, which will be stored on a password-protected local area network drive, accessible only by the Research Officer and the chief investigators. On completion of data analysis and report writing, computer files will be transferred to CD-ROM, which will then be stored in a locked cabinet, accessible only by the Research Officer and the chief investigators.

Data in paper format will be stored for 7 years, while computer files will be stored for 15 years. All will be shredded by a contracted security waste disposal company at the conclusion of the storage period.

\section{Statistical methods}

\section{Analysis of primary and secondary outcomes}

This evaluation will focus on the impact and acceptability of the new model of care, with system-level outcomes being of primary interest. Cross-sectional data will be collected at baseline, 12 and 24 months on the proportion of patients (1) who are screened for distress at least once and (2) who have a psychosocial care plan developed.

Data will be collected at the three time points and analysed using Poisson or Negative Binomial regression depending on overdispersion of the counts of each outcome. The Poisson/Negative binomial models will include a time variable ( 0,12 and 24 months), a before and after variable, a term for the interaction of these two variables and an offset variable which is used to adjust for the total number of patients consented at each time point. The interaction term will be used to estimate any difference between the two periods, which would indicate a slow improvement in the outcome during the intervention period. The before and after variable will be used to estimate the change in the outcome that may occur immediately after the introduction of the model of care. The models will adjust as appropriate for potential confounding variables such as age, gender, residential location, indigenous status, cancer type and time since diagnosis.

\section{Patient experience and health professional knowledge and} confidence

For patient experience surveys, a linear regression will be used to model the patient experience scores over the 
three time points to determine whether there was a significant improvement during the study period while adjusting for potential confounding variables (eg, duration of care within the service, other psychosocial support services used, disease and treatment characteristics, age, and gender). Similarly, for the health professional knowledge and confidence surveys, a linear regression will be used to determine whether there was a difference in the knowledge/confidence scores between the two time points while adjusting for potential confounding variables (eg, other training in psychosocial care received, duration of employment in this service, prior experience, work role and time allocation, age, and gender).

\section{Economic analysis}

Estimates of resource use and costs will take into account the costs of implementation of the new model, but will not include costs of the evaluation/audit. At each time point (baseline, 12 and 24 months), mean estimates of costs will be used and CIs will be generated by boot-strapping the data. Benefits will be measured via surveys and interviews to ascertain the acceptability of the intervention and how the new model is experienced, as well as changes in clinicians' knowledge and confidence. Costs and outcomes will be reported separately at each time point and trends over time will be evaluated.

\section{Qualitative analysis of HCP interviews}

HCP interviews will be audiorecorded, transcribed verbatim and analysed qualitatively. Inductive thematic analysis will be used to identify, analyse and report themes (or patterns) in the data (MapInfo Professional V.8, MapInfo Corporation). Transcripts will be initially read and any words, statements and/or paragraphs related to HCPs' views on the PACT intervention will be extracted by assigning a label or code. Similar excerpts will be identified by using the same code, with clustering of the codes denoting themes in the data. Data analysis will focus on a detailed description of emerging themes, with a focus on identifying the positive and negative aspects of the PACT model and strategies to support its ongoing implementation in the care facility.

\section{DISCUSSION}

This research programme specifically addresses the objective of improving quality of care of patients with cancer and has been developed to address the existing gaps in psychosocial care. The proposed programme will provide a mechanism for delivering integrated, high quality, evidence-based cancer care that is embedded in routine practice, and responsive to the needs of individual patients with cancer by (1) systematically identifying patients' physical and psychosocial health needs, (2) developing care pathways and plans to address identified needs, (3) linking patients to skilled HCPs and appropriate services and (4) coordinating ongoing psychosocial healthcare. The project has a focus on translating evidence regarding psychosocial care into an integrated model that promotes the role of 'frontline' clinical staff, including those in rural settings, and will promote improved coordination and continuity of care for patients in rural and remote areas. Improving the psychosocial component of routine care, building distress screening into a model of routine care and developing a psychosocial care plan for patients will enhance the acceptability and appropriateness of psychosocial care. Specialist services will be active in providing training, advice and support within an interprofessional team, and providing specialist assessment and treatment as a member of this team when needed. Addressing the process and outcome variables will support investigation of the quality of care provided from the patient's and clinician's perspectives.

The proposed care model has the potential to address several unmet needs identified in key policy documents and surveys. Specifically, it will provide improved emotional support and information for cancer inpatients and outpatients, ${ }^{9}$ expand psycho-oncology services to improve access to specialised care ${ }^{19}$ and provide timely individualised support to the level and detail required for patients. ${ }^{32}$ In addition, this research programme directly addresses the priority issue of delivering quality cancer care that addresses patients' physical and psychosocial health needs ${ }^{6} 8$ by bringing together the scientific evidence about the management of psychosocial problems of patients with cancer into a model of patient-centred cancer care. Furthermore, it focuses on patients with urological or H\&N cancers given their lack of research attention compared with their burden on the Australian community. ${ }^{33}$

The translational capacity of this research programme is enhanced through three major strengths. First, it is supported by a very strong collaborative team. Enhancing the role of 'frontline' clinical staff, defining pathways of care and promoting integration between major centres and rural clinicians entail a high level of support from clinical staff for objective implementation and evaluation. This project has a strong support from the highest level of cancer service governance in our area, senior nursing clinicians and existing on-site psychiatric services. The linkage of the PACT model to arealevel network of psycho-oncology services will promote integration of psychosocial care into routine clinical care, promote continuity of care and, through its clinician training model, improve the overall quality of care for patients and their families.

Second, the study utilises a strong methodology appropriate for health services research. The evaluation framework and methodology ensure that the evaluation is sensitive to the role of general clinical staff, appropriate to the setting, relevant to stakeholders, inclusive and informative. Cost analyses will be undertaken to facilitate comparisons of costs and outcomes and changes in these over time; an important consideration as economics is an 
often overlooked element of interventional research. The close engagement of clinicians in the planning, implementation and evaluation will ensure a maximum relevance of the project to the local context of clinical practice, including rural and remote settings. The built-in capacity for flexibility in the clinical setting (eg, documenting and addressing local barriers to integration of psychosocial care) will promote translation to routine care, and potential applicability to other settings. ${ }^{34}$

Third, there is a strong support for integration of psychosocial care into routine care, and efficient use of specialist services. This project will provide important evidence for the effective use of existing resources for nurses and allied health professionals working in routine cancer care. If the outcomes of the research are positive, this will provide the basis for a model of implementing psycho-oncology services across other clinical services within this network. While the project includes the use of routine screening, it does so in a manner that builds this into a model of care, so that these tools can support clinical practice within a model of integrated care, defined service pathways and support to distant sites. The work has substantial potential for translation into other cancer services beyond the research collaborators.

Despite the strengths, there are also several challenges for the research and evaluation. The target population is vulnerable and experiencing an acute stressor that may impact on recruitment. As the intervention will continue over a 24-month period, changes in health professional staff over that time may be substantial. Hence, some of the health professionals completing the survey at 24 months may have been minimally exposed to the intervention, hence potentially diluting the perceptions of impact. Finally, health initiatives introduced more broadly into hospitals in the study location may affect the impact of this intervention, but are beyond the control of the research team.

\section{ETHICS AND DISSEMINATION}

Minor adverse events (eg, a participant being tearful and distressed when talking with the Research Officer) will be logged and fed back to the study team by the end of the study. Serious adverse events (eg, expressing suicidal thoughts) will be reported immediately to the chief investigators and to the ethics committees. Any protocol amendments will be submitted to the ethics committees before these are implemented, and relevant changes will also be communicated to other relevant organisations (eg, trial registry).

\section{Confidentiality}

The names of potential patient and HCP participants will be entered into the study's password-protected administrative database, accessible only by the Research Officer. On receipt of completed surveys and/or consent forms from participants, a study ID will be assigned to each participant, and recorded with identifying information only in the study's password-protected administrative database. Thereafter, survey, consent form and request for results data will be linked to participants only via the allocated study ID. There is no foreseeable reason for personal or identifying participant information to be shared throughout the conduct of the trial, except where required for adverse event reporting.

All of the electronically stored personal participant information will be maintained and destroyed in the same manner as for all data collected throughout the study. On completion of data analysis and report writing, computer files will be transferred to CD-ROM, which will then be stored in a locked cabinet, accessible only by the Research Officer. These will be stored for 15 years, then shredded by a contracted security waste disposal company at the conclusion of the storage period.

\section{Access to data}

The study's chief investigators (AG and BK), Research Officer, Biostatistician and Health Economists will have exclusive access to the final trial dataset.

\section{Dissemination policy}

The results will be widely disseminated through peerreviewed publications as well as relevant HCP and consumer publications. Oncology HCPs and administrators within HNELHD will be invited to a face-to-face presentation of the results by the chief investigators. Presentations will be delivered at relevant national oncology and nursing conferences and meetings. The results will be reported to the funding body and other peak bodies with influence on cancer policy and practice, including Cancer Australia, Clinical Oncology Society of Australia, Cancer Council Australia and Cancer Voices Australia. In addition, a copy of the summary of the study's key findings will be mailed to all research participants who request one, on completion of the project.

\section{Author affiliations}

${ }^{1}$ Ingham Institute for Applied Medical Research, South Western Sydney Clinical School, UNSW Medicine, The University of New South Wales,

Liverpool, New South Wales, Australia

${ }^{2}$ Priority Research Centre for Translational Neuroscience and Mental Health, School of Medicine and Public Health, The University of Newcastle, Callaghan, New South Wales, Australia

${ }^{3}$ Consultation Liaison Psychiatry Service, Hunter New England Local Health District, John Hunter Hospital (JHH), New Lambton, New South Wales, Australia

${ }^{4}$ Priority Research Centre for Health Behaviour \& Hunter Medical Research Institute, The University of Newcastle, Callaghan, New South Wales, Australia ${ }^{5}$ Centre for Health Economics Research \& Evaluation, University of Technology Sydney, Ultimo, New South Wales, Australia

${ }^{6}$ Hunter New England Cancer Network Directorate, Hunter New England Local Health District, JHH, New Lambton, New South Wales, Australia

${ }^{7}$ Hunter New England Cancer Services, Hunter New England Local Health District, JHH, New Lambton, New South Wales, Australia

Acknowledgements The study detailed in this protocol is endorsed by the Psycho-oncology Co-operative Research Group (PoCoG), The University of Sydney, Australia. The study protocol and relevant documents have been reviewed by the PoCoG Scientific Advisory Committee and the Joint 
Community Advisory Group. Debbie 0'Brien and Gai Shylan will play a central role in facilitating recruitment and implementation of the intervention, and Catherine Adams and Deanna Sue in the communication skills training of health professionals involved in the delivery of the PACT model of care.

Contributors $B K$ and $A G$ conceived of the study and are the grant holders. $A B$ assisted in the initial study design and $A P$ and $D B$ provided organisational guidance on service model and implementation. BK and AG, along with the newly appointed clinical staff, developed the communication skills training, and $\mathrm{HC}$ will oversee the day-to-day study implementation according to the protocol. MH and RV provided guidance on data requirements for the economic analysis and will undertake these analyses. JD provided guidance on sample size requirements and will be conducting the primary statistical analyses. All authors contributed to refinement of the study protocol and approved the final manuscript.

Funding This work is funded by the Cancer Institute New South Wales (NSW), grant number 10/THS/2-08.

Competing interests As a Consultant Psychiatrist for the Consultation Liaison Psychiatry Service of John Hunter Hospital and a chief investigator on this project, BK may experience competing interests with respect to reporting the impact, cost and acceptability of the new psychosocial care model. These potentially competing interests will be minimised and managed by having the other chief investigator, AG, primarily responsible for the conduct of the research and result reporting.

Patient consent Obtained.

Ethics approval Hunter New England Human Research Ethics Committee and The University of New South Wales Human Research Ethics Committee.

Provenance and peer review Not commissioned; externally peer reviewed.

Open Access This is an Open Access article distributed in accordance with the Creative Commons Attribution Non Commercial (CC BY-NC 3.0) license, which permits others to distribute, remix, adapt, build upon this work noncommercially, and license their derivative works on different terms, provided the original work is properly cited and the use is non-commercial. See: http:// creativecommons.org/licenses/by-nc/3.0/

\section{REFERENCES}

1. Eton DT, Lepore SJ. Prostate cancer and health-related quality of life: a review of the literature. Psychooncology 2002;11:307-26.

2. Ledeboer QCP, van der Velden LA, de Boer MF, et al. Physical and psychosocial correlates of head and neck cancer: an update of the literature and challenges for the future (1996-2003). Clin Otolaryngol 2005;30:303-19.

3. Vickery LE, Latchford G, Hewison J, et al. The impact of head and neck cancer and facial disfigurement on the quality of life of patients and their partners. Head Neck 2003;25:289-96.

4. Smith KB, Humphreys JS, Wilson MGA. Addressing the health disadvantage of rural populations: how does epidemiological evidence inform rural health policies and research? Aust J Rural Health 2008;16:56-66.

5. Underhill CR, Goldstein D, Grogan PB. Inequity in rural cancer survival in Australia is not an insurmountable problem [editorial]. Med J Aust 2006;185:479-80.

6. National Breast Cancer Centre and National Cancer Control Initiative. Clinical practice guidelines for the psychosocial care of adults with cancer. Camperdown: National Breast Cancer Centre, 2003.

7. Sharpe M, Strong V, Allen K, et al. Major depression in outpatients attending a regional cancer centre: screening and unmet treatment needs. Br J Cancer 2004;90:314-20.

8. Institute of Medicine. Cancer care for the whole patient: meeting psychosocial health needs. Washington: The National Academies Press, 2008

9. Cancer Institute NSW. New South Wales cancer patient satisfaction survey 2008. Sydney: Cancer Institute NSW, 2009.
10. Department of Health. National cancer patient experience survey 2011/12-National Report. 2012. http://www.dh.gov.uk (Search for: 17827).

11. Canadian Partnership Against Cancer. The 2012 cancer system performance report. Toronto: Canadian Partnership Against Cancer, 2012.

12. Holland JC, Kelly BJ, Weinberger MI. Why psychosocial care is difficult to integrate into routine cancer care: stigma is the elephant in the room [editorial]. J Natl Compr Canc Netw 2010;8:362-6.

13. Hutchison SD, Steginga SK, Dunn J. The tiered model of psychosocial intervention in cancer: a community based approach. Psychooncology 2006;15:541-6.

14. Strong V, Waters $\mathrm{R}$, Hibberd $\mathrm{C}$, et al. Management of depression for people with cancer (SMaRT oncology 1): a randomised trial. Lancet 2008;372:40-8.

15. Loscalzo M, Clark KL, Holland J. Successful strategies for implementing biopsychosocial screening [commentary]. Psychooncology 2011;20:455-62.

16. Forsythe LP, Rowland $\mathrm{JH}$, Padgett $\mathrm{L}$, et al. The cancer psychosocial care matrix: a community-derived evaluative tool for designing quality psychosocial cancer care delivery. Psychooncology 2013;22:1953-62.

17. Sanson-Fisher RW, Bonevski B, Green LW, et al. Limitations of the randomized controlled trial in evaluating population-based health interventions. Am J Prev Med 2007;33:155-61.

18. Reeves BC, Deeks JJ, Higgins JPT, et al. Chapter 13: including non-randomized studies. In: Higgins JPT, Green S. eds. Cochrane handbook for systematic reviews of interventions. Version 5.0.1. West Sussex: John Wiley \& Sons Ltd, 2008:391-432.

19. Hunter New England Area Health Service. HNE health area cancer services plan 2006-2010. New Lambton: Hunter New England Area Health Service, 2006.

20. Butow P, Cockburn J, Girgis A, et al. Increasing oncologists' skills in eliciting and responding to emotional cues: evaluation of a communication skills training program. Psychooncology 2008;17:209-18.

21. Girgis A, Cockburn J, Butow $P$, et al. Improving patient emotional functioning and psychological morbidity: evaluation of a consultation skills training program for oncologists. Patient Educ Couns 2009;77:456-62.

22. National Comprehensive Cancer Network. NCCN clinical practice guidelines in oncology: distress management. Version 2.2013. FortWashington: National Comprehensive Cancer Network, 2012.

23. Tuinman MA, Gazendam-Donofrio SM, Hoekstra-Weebers JE. Screening and referral for psychosocial distress in oncologic practice: use of the distress thermometer. Cancer 2008;113:870-8.

24. Feder G, Eccles M, Grol R, et al. Using clinical guidelines [clinical guidelines]. BMJ 1999;318:728-30.

25. NRC+Picker. NSW health: patient survey 2007-cancer care survey. Lincoln: NRC+Picker, 2007.

26. NRC+Picker. NSW health: patient survey 2007-hospital careovernight patient survey. Lincoln: NRC+Picker, 2007.

27. Cancer Council Victoria. Critical cancer care events scale. Carlton: Cancer Council Victoria, 2007.

28. Clayton JM, Butow PN, Waters A, et al. Evaluation of a novel individualised communication-skills training intervention to improve doctors' confidence and skills in end-of-life communication. Palliat Med 2013;27:236-43

29. Dodson S. Client-centred care-training needs survey. Version 2 Melbourne: Eastern HARP, 2009.

30. Ashworth CD, Williamson P, Montano D. A scale to measure physician beliefs about psychosocial aspects of patient care. Soc Sci Med 1984;19:1235-8.

31. Schmoldt RA, Freeborn DK, Klevit HD. Physician burnout: recommendations for HMO managers. HMO Pract 1994;8:58-63.

32. Cancer Institute NSW. NSW cancer plan 2007-2010. Sydney: Cancer Institute NSW, 2006.

33. Cancer Australia. Cancer research in Australia: an overview of cancer research projects and research programs in Australia 2003 to 2005. Sydney: Cancer Australia, 2007.

34. Medical Research Council. Developing and evaluating complex interventions: new guidance. 2008. http://www.mrc.ac.uk/ complexinterventionsguidance (accessed Aug 2013). 